\title{
Comparative genomics of plant pathogenic Botrytis species with distinct host specificity
}

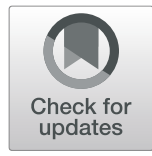

\author{
Claudio A. Valero-Jiménez ${ }^{1}$, Javier Veloso ${ }^{1,2}$, Martijn Staats ${ }^{3,4}$ and Jan A. L. van Kan ${ }^{1 *}$ (D)
}

\begin{abstract}
Background: Fungi of the genus Botrytis (presently containing $\sim 35$ species) are able to infect more than 1400 different plant species and cause losses in a wide range of crops of economic importance. The best studied species is $B$. cinerea, which has a broad host range and is one of the best studied necrotrophic plant pathogenic fungi. Most other Botrytis spp. have a narrow host range and have been studied in less detail. To characterize genomic variation among different representatives of Botrytis spp., we sequenced and annotated the draft genomes of nine Botrytis species: B. calthae, B. convoluta, B. elliptica, B. galanthina, B. hyacinthi, B. narcissicola, B. paeoniae, B. porri and B. tulipae.

Results: Bioinformatics and comparative genomics tools were applied to determine a core of 7668 shared protein families in all Botrytis species, which grouped them in two distinct phylogenetic clades. The secretome of all nine Botrytis spp. was similar in number (ranging from 716 to 784 predicted proteins). A detailed analysis of the molecular functions of the secretome revealed that shared activities were highly similar. Orthologs to effectors functionally studied in B. cinerea were also present in the other Botrytis species. A complex pattern of presence/absence of secondary metabolite biosynthetic key enzymes was observed.

Conclusions: Comparative genomics of Botrytis show that overall, species share the main signatures and protein families in the secreted proteins, and of known effectors. Our study provides leads to study host range determinants in the genus Botrytis and provides a stepping stone to elucidate the roles of effector candidates in the infection process of these species.
\end{abstract}

Keywords: Effector, Grey mould, Necrotroph, Secondary metabolite, Secretome

\section{Background}

The fungal genus Botrytis comprises $~ 35$ species, which all interact with plants $[1,2]$. With one exception, they are notorious pathogens with a necrotrophic infection behaviour, i.e. they kill host cells and invade the dead cells to acquire nutrients. The single exception, $B$. deweyae, can grow as a symptomless endophyte inside its host plant Hemerocallis, and occasionally switches lifestyle into a necrotizing infection destroying the young foliage, thereby causing 'spring sickness' [3]. The two most extensively studied species with a necrotrophic lifestyle, B. cinerea and B. pseudocinerea, are morphologically indistinguishable and cause grey mould on a broad range of (>1400) host plant species [4]. Other Botrytis species are considered to be restricted to a single host species or a

\footnotetext{
* Correspondence: jan.vankan@wur.nl

${ }^{1}$ Laboratory of Phytopathology, Wageningen University, 6708PB Wageningen, the Netherlands

Full list of author information is available at the end of the article
}

small number of taxonomically related hosts [5]. Phylogenetic analysis has separated the genus Botrytis into two distinct clades, one of which comprises $B$. cinerea and $B$. pseudocinerea along with a small number of species pathogenic on dicot plants, while the second (large) clade comprises mainly species pathogenic on monocot host plants, and especially on bulb-producing monocot species $[1,2,5]$. Many ornamental bulb flower crops of economic relevance (tulip, lily, Narcissus, snowdrop, Gladiolus) are each infected by a separate specialized Botrytis species [5].

Fungi with a necrotrophic lifestyle achieve plant cell death by the induction of an apoptotic programmed cell death in the host [6]. Rather than brutally killing the host cells, these pathogens co-opt the plant cell death machinery to their own benefit. Evidence is accumulating that many plant pathogenic fungi with a necrotrophic lifestyle secrete effector molecules (proteins and/ or secondary metabolites) that trigger the host cell death machinery, following effector recognition by a cognate 
receptor protein in the host plant [7]. In interactions of Parastagonospora nodorum with its host (wheat), several cases have been described of inverse gene-for-gene interactions, in which a $P$. nodorum effector protein genetically interacts with a single genetic locus in the host that confers response to the effector and susceptibility to a fungal isolate producing this effector [8]. The cyclic non-ribosomal peptide victorin from Cochliobolus victoriae also triggers apoptotic programmed cell death in plants carrying the genetic locus for sensitivity $[9,10]$.

Several plant cell death-inducing proteins and metabolites were identified in B. cinerea [11-14], all of which act indiscriminately on all dicot plants that were tested. None of these necrotrophic effectors was essential for virulence of $B$. cinerea and none of them is considered to play a role in host specificity. Yet, there is evidence for the production of necrotrophic effectors in some specialised Botrytis species. The lily pathogen B. elliptica produced in liquid culture a proteinaceous effector activity that caused apoptotic cell death exclusively in lily, and conferred upon the non-pathogen $B$. cinerea the capacity to infect lily following effector infiltration [15]. Also the broad bean pathogen $B$. fabae was reported to produce a host-specific effector activity [16]. In order to understand the mechanisms of infection of host-specific Botrytis species, as compared to the broad host range pathogen B. cinerea, we generated draft genome sequences of nine Botrytis species, mostly selected from the phylogenetic clade of monocot-infecting pathogens, with emphasis on species infecting ornamental flower bulb crops. The genomes were analysed with special attention for the presence of genes that potentially contribute to the host specificity, such as genes encoding effector proteins, enzymes involved in secondary metabolite biosynthesis or enzymes involved in the degradation of monocot versus dicot cell wall polysaccharides.

\section{Results and discussion}

\section{Sequencing and assembly}

The genome assembly sizes of the nine Botrytis species ranged from $43 \mathrm{Mb}$ to $55 \mathrm{Mb}$ (Table 1). The genomes of eight species are similar in size to the previously described genome of $B$. cinerea (43.5 Mb; [17]), while the genome of $B$. narcissicola was $\sim 10 \mathrm{Mb}$ larger than the other species analysed here. Contigs that contained mitochondrial genes were identified with Blast and removed from the assemblies. To estimate the completeness of the assembled genomes, Benchmarking Universal Single-Copy Orthologs (BUSCO) was used. This analysis indicated that all genomes had a high level of completeness (96.3-99.2\%), with the B. elliptica genome being the most complete. The assemblies are predicted to encode between 12,033 and 12,663 protein-coding genes.

\section{Phylogenetics and phylogenomics}

A phylogenetic tree was constructed of 7668 conserved core genes based on the amino acid alignment of $~ 3.7$ million positions, and using Sclerotinia sclerotiorum as outgroup (Fig. 1). The relationship among the Botrytis species is in accordance with previous studies by [5], which divided the genus in two clades based on three protein-coding genes (G3PDH, HSP60 and RPB2). Clade 1 contains species that mainly infect eudicot plants, while species in Clade 2 infect either eudicot or monocot plants (but mainly monocots). A pan-genome analysis for the 9 Botrytis species sequenced in this study in combination with the previously sequenced $B$. cinerea B05.10 [17], indicated that the core genome of Botrytis spp. consists of 7617 orthologous gene clusters (Fig. 2a). On the other hand, the pan-genome consists of 12,245 orthologous gene clusters (Fig. 2b).

\section{GC content distribution}

Previous studies have revealed that some fungal plant pathogens have distinct patterns in their genome, a so called two-speed genome, which consist of an alternation of repeat-rich and gene sparse regions with GC-equilibrated and gene dense regions [18, 19]. Moreover, it has been reported that repeat-rich regions can display an enrichment of rapidly evolving genes [20]. Analysis of the GC content in Botrytis spp. showed the presence of a bimodal distribution of GC content. The proportion of AT-rich regions in the genomes ranged from $4.86 \%$ in B. cinerea up to $27 \%$ in $B$. narcissicola (Table 2). The difference in genome sizes of the nine species, as compared to $B$. cinerea, is correlated with the proportion of AT-rich regions, as shown in B. narcissicola, the largest among the Botrytis spp. sequenced up to date (Table 2). Illumina-based sequence assemblies tend to lack repeats, especially AT-rich repeats [21]. Therefore it can be assumed that a more complete assembly of the nine genomes (based on long read technology) would have an even higher content of AT-rich repeats. Interestingly, $B$. cinerea has the lowest proportion of AT-rich regions in its genome even though it was sequenced with long read technology, which would encompass more difficult to assemble regions such as repetitive regions.

The number of genes located in these AT-rich regions differed per species, with $B$. calthae having the highest number of genes (99) and B. cinerea the lowest (0). In order to elucidate if the AT-rich regions resulted from Repeat-Induced Point mutation (RIP), a meiosis-specific mechanism that affects duplicated sequence regions $[22,23]$, we compared the frequencies of dinucleotides between AT-rich regions and GC-equilibrated regions (Additional file 1). The AT-rich regions contained a strongly elevated frequency of TpA, which is the primary product of RIP in fungi, and is a strong indicator of RIP activity in these genomes [22]. Furthermore, RIP 
Table 1 Assembly and gene prediction information of Botrytis spp. genomes

\begin{tabular}{lllllllll}
\hline Species & Scaffolds & Assembly Size & Largest Scaffold & N50 & BUSCO complete/partial & Predicted genes & $\begin{array}{c}\text { Secretome size } \\
\text { \% of secreted } \\
\text { proteins }\end{array}$ \\
\hline B. calthae & 3985 & $47.56 \mathrm{Mb}$ & 293,700 & 56,053 & $97.5(98.5)$ & 12,492 & 745 & 5.96 \\
B. convoluta & 2054 & $45.40 \mathrm{Mb}$ & 436,056 & 97,955 & $98.7(99.1)$ & 12,532 & 752 & 6.00 \\
B. elliptica & 5594 & $47.68 \mathrm{Mb}$ & 230,072 & 36,976 & $99.2(99.3)$ & 12,663 & 762 & 6.02 \\
B. galanthina & 3422 & $43.97 \mathrm{Mb}$ & 354,402 & 65,043 & $98.9(99.0)$ & 12,575 & 784 & 6.23 \\
B. hyacinthi & 2509 & $43.91 \mathrm{Mb}$ & 532,180 & 115,520 & $99.0(99.2)$ & 12,197 & 752 & 6.17 \\
B. narcissicola & 8392 & $54.85 \mathrm{Mb}$ & 289,279 & 43,203 & $96.3(98.1)$ & 12,341 & 730 & 5.92 \\
B. paeoniae & 1833 & $46.36 \mathrm{Mb}$ & 901,367 & 125,168 & $99.0(99.2)$ & 12,138 & 746 & 6.15 \\
B. porri & 4737 & $43.11 \mathrm{Mb}$ & 181,158 & 35,774 & $97.4(97.9)$ & 12,033 & 716 & 5.95 \\
B. tulipae & 653 & $45.45 \mathrm{Mb}$ & 738,496 & 172,411 & $98.8(99.0)$ & 12,656 & 750 & 5.93
\end{tabular}

indices (ratio of TpA/ApT dinucleotide frequency) indicate RIP activity in all Botrytis spp. analysed here (Additional file 2).

\section{Secretome functions and effector proteins}

The secretome of all nine Botrytis spp. was similar in number (ranging from 716 proteins in $B$. porri to 784 in B. galanthina), representing 5 to $6 \%$ of the total proteome (Table 1), in line with what was reported for $B$. cinerea [17, 24]. The molecular function of the secreted proteins could be annotated for more than $50 \%$ of the secretome of each species (Fig. 3). There were no significant differences between the species in any of the Gene Ontology categories (error bars in Fig. 3 denote variability between species). Hydrolase activity (GO:0016787) was the most common molecular function of the secretome for all Botrytis species (around 25\% of the total secretome), similar to what was reported previously for $B$. cinerea [25]. B. galanthina, B. hyacinthi and B. cinerea have the highest number of genes with hydrolase activity (approx. 230), while B. porri and B. calthae have the lowest number (approx. 200 genes). Half of the genes with hydrolase activity are plant cell wall degrading enzymes (PCWDEs, Table 3) with a potential role in host tissue decomposition and pathogenesis. Oxidoreductase activity (GO:0016491) was present in the secretome of all Botrytis species, representing $10 \%$ of the total secretome. This activity was more abundant in $B$. galanthina, $B$. cinerea and $B$. tulipae with approx. 70 proteins, while it was less abundant in B. porri (56 proteins). Transferase activity

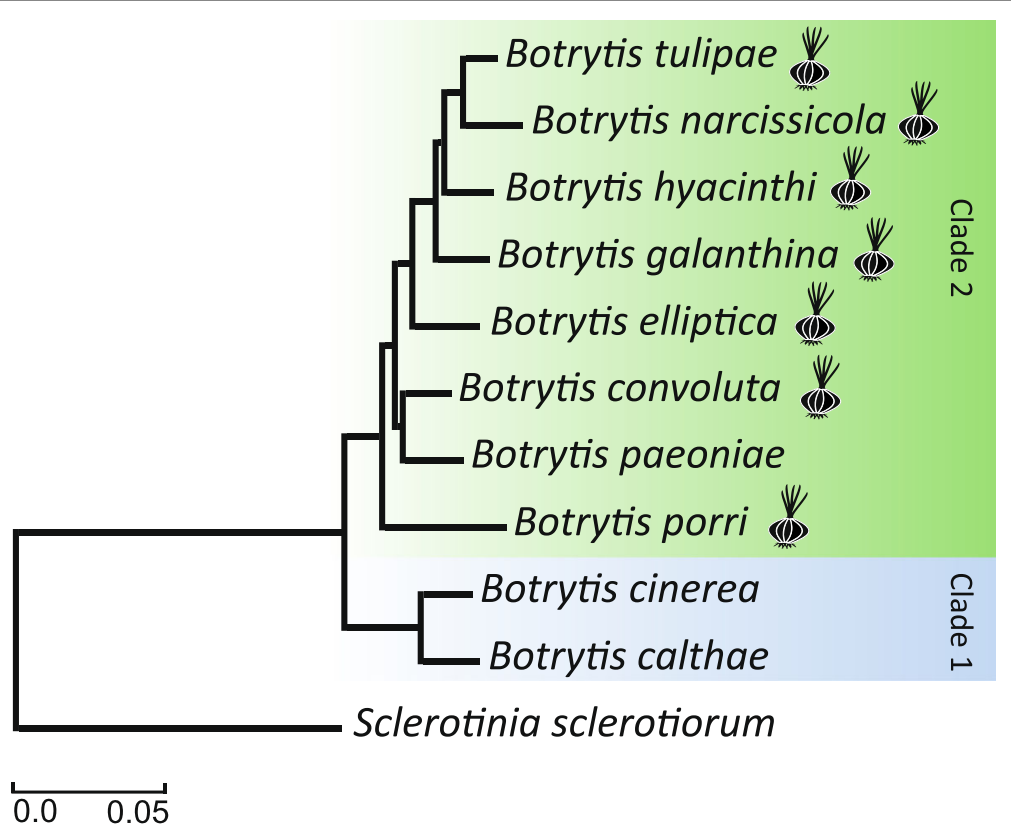

Fig. 1 Phylogenetic tree based on single-copy orthologous genes of different Botrytis species, with S. sclerotiorum as the outgroup to root the tree. All branches have a high bootstrap support (ML > 90). Two clades previously reported in the genus Botrytis are highlighted. The bulb plant symbols next to the species names indicate species that infect monocotyledonous plants, species without the symbol infect dicotyledonous host plants 

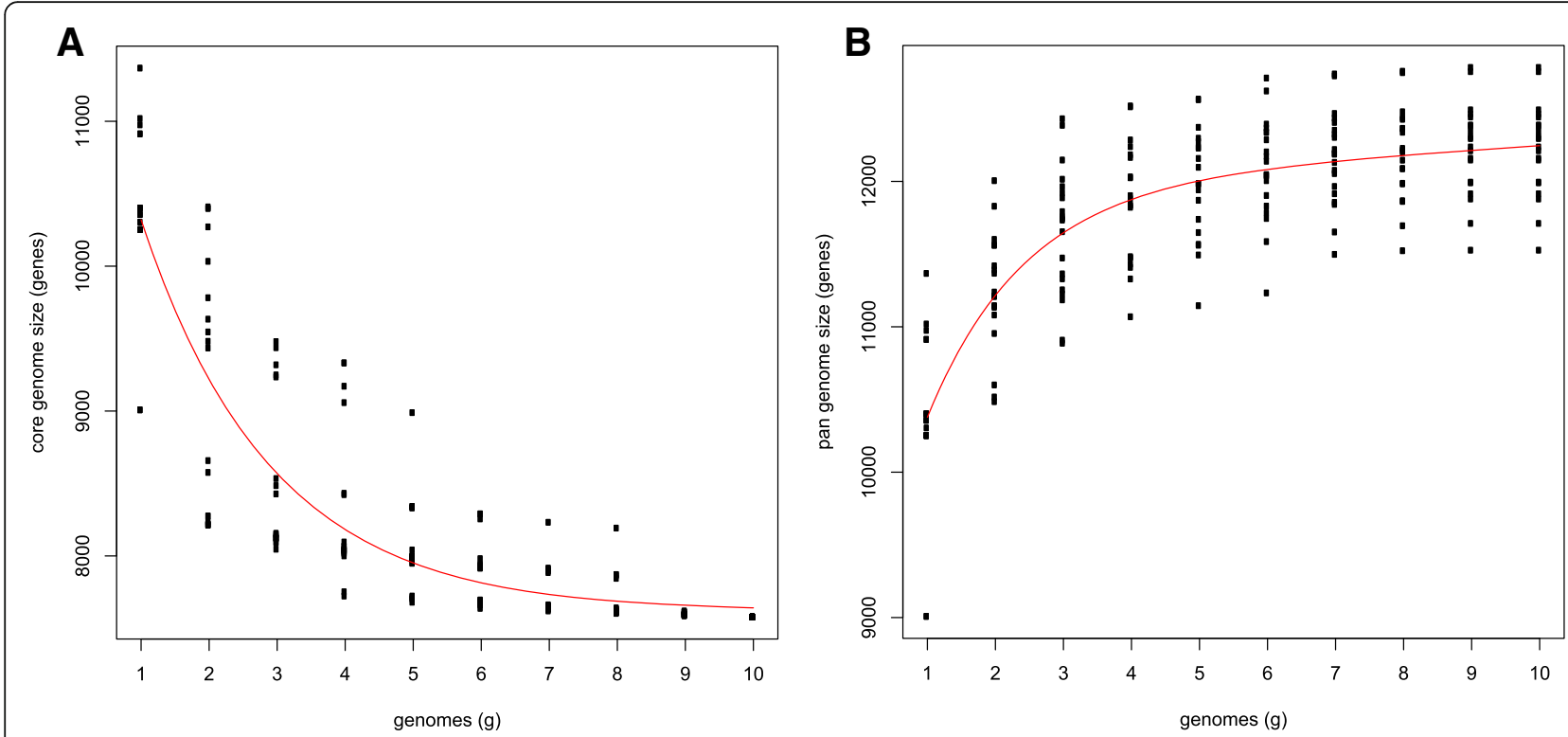

Fig. 2 Pan-genome analysis of Botrytis spp. a Estimation of the Botrytis spp. core genome, in which the number of shared genes is plotted as a function of the number of species sequentially added. $\mathbf{b}$ Estimation of Botrytis spp. pan-genome size, in which the numbers of all genes are plotted as a function of the number of species sequentially added.

(GO:0016740) was present in similar levels as the oxidoreductase activity ( $10 \%$ of the total secretome) and B. porri was among the species with a higher number of proteins (approx. 70) along with B. elliptica and B. cinerea, while B. convoluta and $B$. calthae had a lower number of proteins with this activity (approx. 60). The final fairly abundant molecular function was isomerase activity (GO:0016853), accounting for $5 \%$ of the total secretome in all the species. The annotations of the secreted proteins in the GO Biological Process domain were mainly related to carbohydrate metabolic processes (GO:0005976, GO:0000272, GO:0044262), cell wall (GO:0071554) and pigment processes (GO:0042440), while annotations in the GO Cellular Component domain were mainly the cell periphery (GO:0071944) and cell wall (GO:0005618), as is expected from secreted proteins (Additional file 3). The 10 Botrytis species did not show significant differences in the number of genes in any of the categories of these GO domains.

All secreted proteins of the nine newly sequenced species and $B$. cinerea were clustered using Orthofinder and out of
7521 proteins, 7189 were assigned to 901 orthologous groups. A total of 393 orthologous groups were shared among all 10 Botrytis species (Fig. 4). Orthologs to known effectors or virulence factors from $B$. cinerea were present in this subset. For instance, the $B c x y n 11 \mathrm{~A}$ gene which encodes a necrosis-inducing xylanase [26], and $B c p g 1$ and $B c p g 2$, encoding endopolygalacturonases that are required for full virulence [27, 28], are present in all Botrytis spp. Furthermore, all Botrytis spp. contained an ortholog to the $B c p l s 1$ gene, which encodes an integral membrane tetraspanin protein that participates in $B$. cinerea appressorium function and is required for full virulence [29]. Orthologs of the $B c s p l 1$ gene, encoding a protein with a cerato-platanin domain that elicits plant defences and is required for full virulence [11] are also present in all Botrytis spp. The nep 1 and nep 2 genes, encoding phytotoxic effector proteins that induce necrosis and the synthesis of ethylene in plant leaves, are also present in all Botrytis spp., as previously reported [30, 31]. There are proteins from other plant pathogenic fungi that are involved in pathogenesis and contain a CFEM domain, which contains eight cysteines

Table 2 GC content distribution among Botrytis spp.

\begin{tabular}{|c|c|c|c|c|c|c|c|c|c|c|}
\hline & BCAL & $\mathrm{BCIN}$ & $\mathrm{BCON}$ & BELL & BGAL & BHYA & BNAR & BPAE & BPOR & BTUL \\
\hline Genome Size & 47.4 & 42.6 & 45.3 & 47.6 & 43.9 & 43.8 & 54.8 & 46.2 & 43 & 45.3 \\
\hline \%AT rich regions & 15.6 & 4.86 & 12.6 & 12.7 & 6.45 & 9.79 & 27 & 15.9 & 7.6 & 9.95 \\
\hline GC peak in AT rich regions & 32.5 & 18.2 & 26.4 & 18.1 & 18.1 & 25 & 27 & 30.5 & 19.7 & 16.2 \\
\hline Genes in AT rich regions & 99 & 0 & 8 & 4 & 1 & 6 & 17 & 33 & 4 & 2 \\
\hline Gene density in AT rich regions & 13.3 & 0 & 1.4 & 0.66 & 0.35 & 1.4 & 1.15 & 4.49 & 1.22 & 0.44 \\
\hline Range of GC content in AT rich regions & $0-37$ & $0-27.4$ & $0-33.6$ & $0-28.6$ & $0-27.7$ & $0-32.6$ & $0-35.4$ & $0-36$ & $0-28.7$ & $0-27.5$ \\
\hline
\end{tabular}




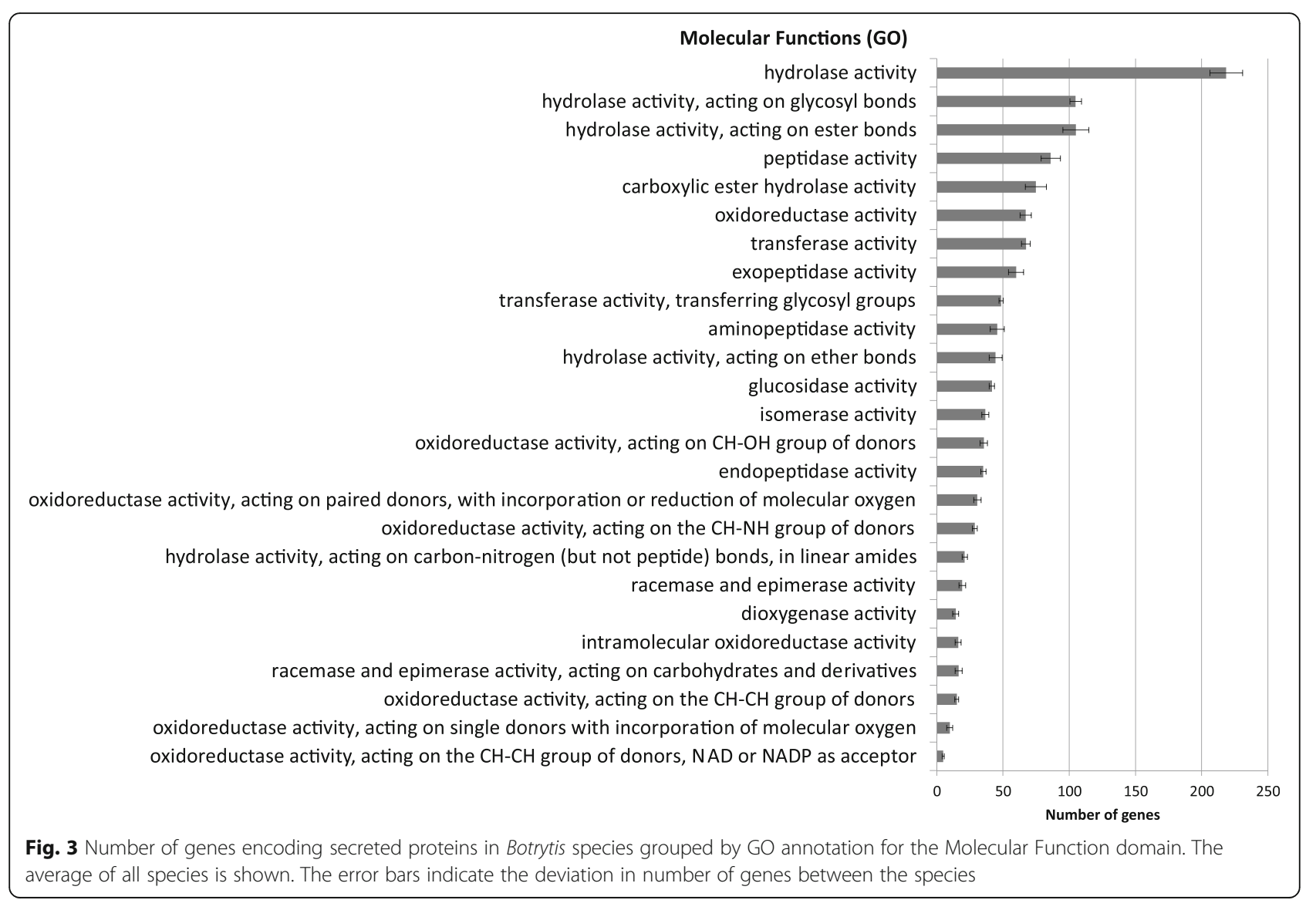

[32-34]. The mechanism by which CFEM domain-containing proteins act is unknown but a recent study reported that $B$. cinerea lacking $B c C F E M 1$ is less virulent [35]. Three orthogroups in the secretome contain a CFEM domain, of which the orthogroup of $B C C F E M 1$ is present in all Botrytis spp., another is shared between $B$. calthae, $B$. cinerea, $B$. elliptica, B. hyacinthi, B. paeoniae and B. porri, and the third orthogroup is only present in $B$. porri. Only the orthogroup shared between all Botrytis spp. is considered an effector based on EffectorP.
Besides the orthogroups shared by all ten Botrytis spp., an additional 332 orthologous groups are unique to only one single species (columns 2-11 in Fig. 4) and 102 groups (columns $12-18$ in Fig. 4) are common to all species but one. Nine orthologous groups are present only in the two species from clade 1 (B. cinerea and $B$. calthae) and seven are present exclusively in the species from clade 2 (last two columns in Fig. 4) [5]. The nine orthologous groups unique to $B$. cinerea and $B$. calthae contain 5 candidate effectors of 9-15 kDa. Additionally,

Table 3 Comparison of plant cell wall degrading enzymes (PCWDEs) among Botrytis spp.

\begin{tabular}{|c|c|c|c|c|c|c|}
\hline Species & Cellulose & Hemicellulose & Hemicellulose or pectin side chains & Pectin & Plant or Fungal CWDEs & Total \\
\hline B. calthae & 17 & 30 & 12 & 32 & 21 & 112 \\
\hline B. cinerea & 20 & 34 & 13 & 38 & 27 & 132 \\
\hline B. convoluta & 18 & 31 & 12 & 34 & 25 & 120 \\
\hline B. elliptica & 19 & 36 & 11 & 35 & 22 & 123 \\
\hline B. galanthina & 19 & 35 & 12 & 36 & 23 & 125 \\
\hline B. hyacinthi & 20 & 34 & 13 & 35 & 23 & 125 \\
\hline B. narcissicola & 20 & 31 & 11 & 28 & 21 & 111 \\
\hline B. paeoniae & 19 & 31 & 12 & 32 & 22 & 116 \\
\hline B. porri & 19 & 30 & 12 & 33 & 22 & 116 \\
\hline B. tulipae & 18 & 32 & 11 & 29 & 22 & 112 \\
\hline
\end{tabular}




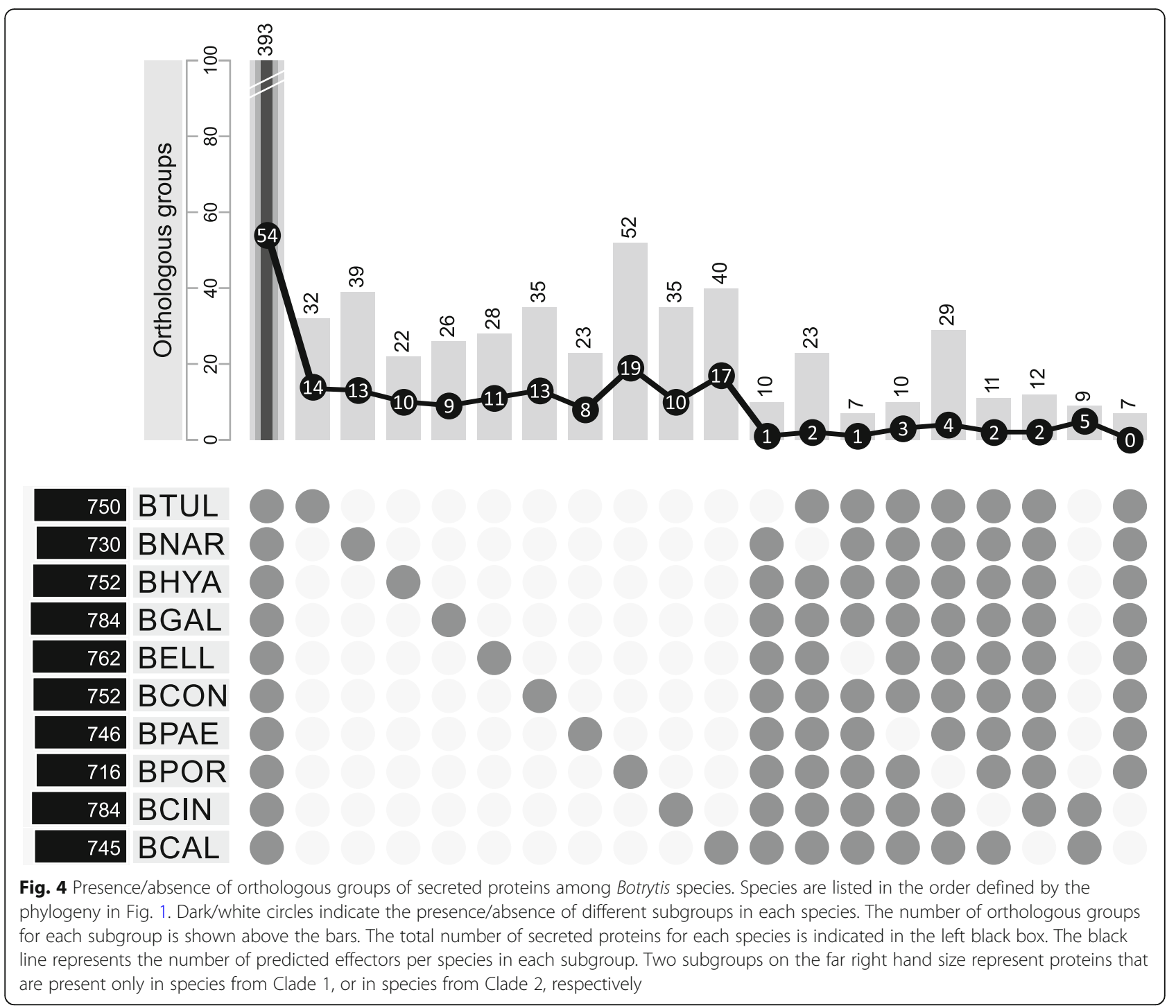

a hydrolase (hydrolyzing O-glycosyl compounds), a protein kinase and an oxidoreductase were present in orthologous groups unique to $B$. cinerea and $B$. calthae. The seven orthologous groups unique to the other eight species contain mainly hydrolytic enzymes (one serine-type peptidase (GO:0008236), one hydrolase acting on glycosyl bonds (GO:0004553), one asparaginase (GO:0004067) and one G1 endopeptidase (GO:0004190)) but no effectors.

In order to elucidate whether putative effector-encoding genes were clustered near repeats, and thus associated with rapidly evolving regions of the genome, we calculated the distances to the nearest repeat for all putative effectorencoding genes and compared them to the distances of a random subset of non-effector-encoding genes to the nearest repeat. Among the 10 species analysed, putative effector genes were on average significantly closer to repeats only in $B$. cinerea and $B$. galanthina but not in the other species (Additional file 4). The biological repercussion of this observation for $B$. cinerea and $B$. galanthina is unclear, however, there is no general tendency in Botrytis of proximity of effector genes to repeats, as was also observed in S. sclerotiorum, a close relative of Botrytis [36].

The orthologous groups of proteins that are unique for one of the Botrytis species are shown in columns 211 of Fig. 4 (for a complete list, see Additional file 5). The different GO molecular functions and effector predictions for these species-specific proteins are shown in Fig. 5. There are 8-19 unique effectors in each species. Whether such species-specific effectors serve as host range determinants requires further functional studies, including the analysis of gene expression during host plant infection, the construction of targeted knockout mutants and the use of effector proteins (produced in heterologous expression systems) in assays for host-specific cell death induction. 


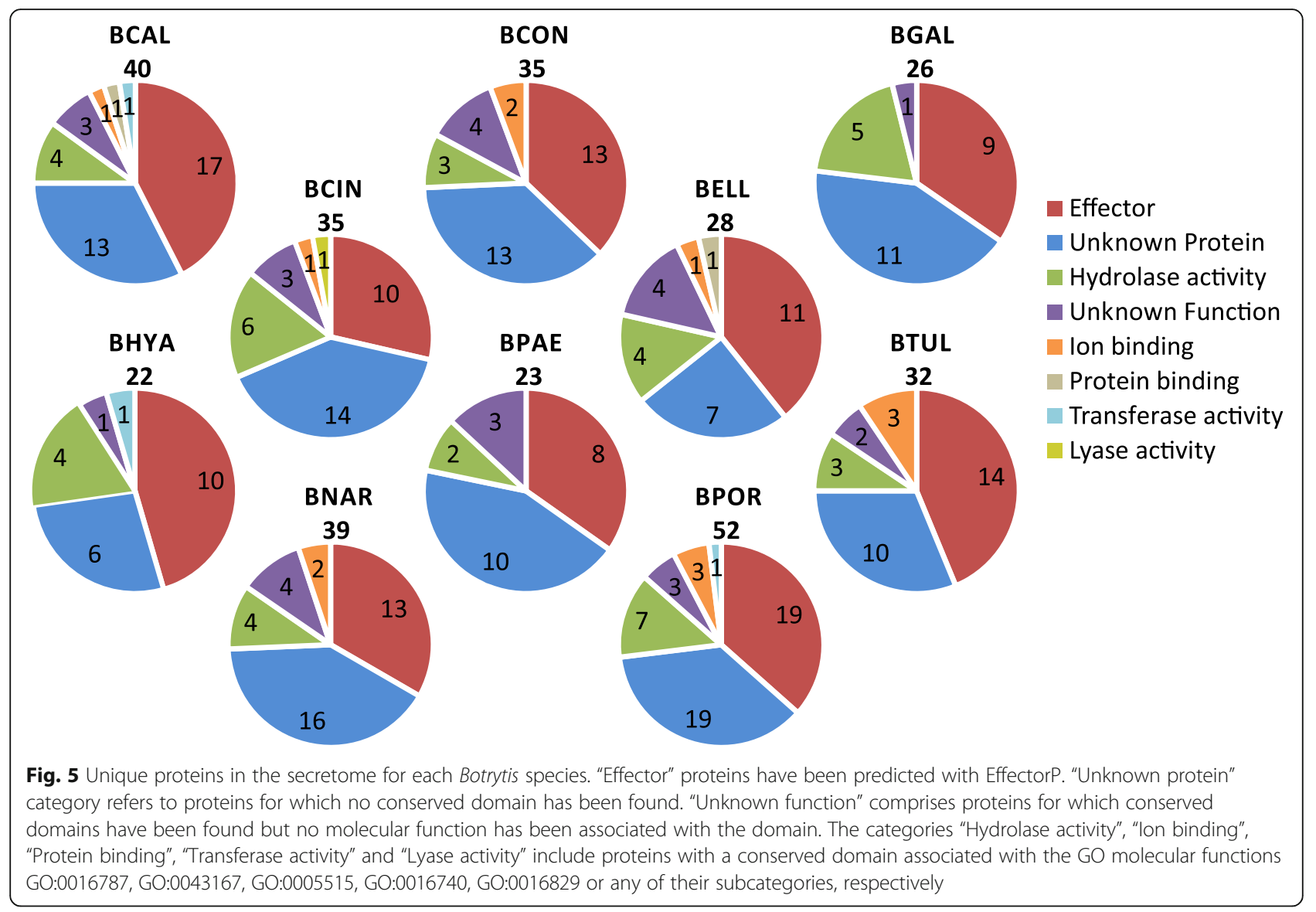

Furthermore, each of the 10 species has its own set of unique hydrolases, however, only few of these hydrolase activities are unique for a single species: only $B$. cinerea possesses a unique lyase and $B$. calthae possesses a unique transferase activity, for which orthologs are undetected in the other nine species. Among the secreted enzymes that are unique to single Botrytis species could be enzymes that detoxify antifungal secondary metabolites produced by host plants as defense against pathogens, collectively known as phytoanticipins or phytoalexins. In order to effectively colonize its host, a pathogenic fungus must be able to inactivate the antifungal compound. Such enzymatic inactivation can involve oxidation or hydrolysis. There is ample evidence for the capacity of several Botrytis species to detoxify host antifungal compounds: $B$. cinerea can degrade resveratrol [37] from grapes and $\alpha$ - tomatine [38] in tomato, B. tulipae degrades tulipalin [39] from tulips, and B. fabae degrades wyerone acid [40] in broad bean. The genes encoding such enzymes have not been identified with the exception of the $B$. cinerea laccase gene $B c l c c 2$ [41]. For most Botrytis species, however, even the phytoalexins in their host plants remain to be chemically characterized, and it remains elusive which types of detoxifying enzymes could play a role in pathogenicity.
We further analysed a subset of the secretome that is related to the degradation of plant cell wall carbohydrates, as this is an important process during host plant infection. The genomes of the nine Botrytis spp. contain between 111 and 125 plant cell wall degrading enzymes (PCWDEs), slightly less than in B. cinerea (132; see Table 3). The PCWDEs can be further subdivided depending on the substrate that they degrade: cellulose, hemicellulose, and pectin. Overall, the number of secreted enzymes capable of degrading cellulose, hemicellulose and pectin is very similar among Botrytis spp., except for $B$. narcissicola and B. tulipae, where the number of pectin degrading enzymes is lower ( 28 and 29 , respectively). The pectin content of plants can differ significantly, especially between monocot and dicot hosts [42]. Despite the fact that seven of the ten Botrytis spp. analysed here infect monocots, the content of PCWDE encoding genes does not differ. This may be correlated with the fact that the monocot hosts of these seven species are members of the Asparagales and Liliales, known to have a relatively high levels of pectin [42] as compared to Gramineae. The large number of genes encoding pectin degrading enzymes in the Botrytis species infecting bulb flower crops therefore should not be considered unusual. 


\section{Secondary metabolite gene clusters}

Fungi are able to produce a wide array of compounds, defined as secondary metabolites (SM), that help them adapt and survive in different environments and compete with other organisms [43]. In B. cinerea, more than 40 secondary metabolite gene clusters have been identified, but only a few of the metabolites have been completely characterized, such as the well-characterized phytotoxins botrydial and botcinic acid (reviewed by [44]). The nine draft genomes were examined for the presence of secondary metabolite clusters present in $B$. cinerea by homology to the $B$. cinerea reference genome (Fig. 6). The two key enzymes related to the production of melanin (Bcpks12 and Bcpks13), a key enzyme related to carotenoid synthesis, retinal (Bcphs1), and a key enzyme putatively involved in synthesis of pyrones, resorcylic acids and resorcinols (Bcchs1), are present in all nine species. Also key enzymes for the synthesis of coprogene siderophore (Bcnrps6) and other siderophores (Bcnrps2, Bcnrps3), that are found across all ascomycetes [45], are shared between Botrytis species. For other SM key enzyme-encoding genes, the distribution was heterogeneous. The key enzymes for the production of botcinic acid (Bcboa6/Bcpks6 and Bcboa9/Bcpks9) turned out to both be present in B. calthae, B. convoluta, B. narcissicola and $B$. porri, whereas B. elliptica, B. galanthina and B. tulipae only contained Bcboa9/Bcpks9 but not Bcboa6/Bcpks6. The SM key enzymes of the botcinic acid cluster were totally absent in B. hyacinthi and $B$. paeoniae. The key enzyme for botrydial synthesis (Bcbot2/Bcstc1) was present in B. elliptica, B. paeoniae and $B$. porri but not in the other species. The key enzyme for production of abscisic acid (Bcaba4) was present in $B$. calthae, B. galanthina, B. narcissicola and B. porri but not in other species.

The remainder of the $B$. cinerea key enzymes are involved in the synthesis of metabolites with an unknown function, but they can be classified based on the chemical nature of the metabolite synthesized by the gene cluster: peptides (non-ribosomal peptide synthetase), polyketides (polyketide synthase), terpenes (terpene synthase) and alkaloids (dimethylallyl tryptophane synthase). Of those, orthologs of some key enzymes are shared between all species (five polyketides, four peptides, and one alkaloid), some are only present in $B$. cinerea (indole-terpene Bcpax1 and peptide-polyketide Bcpks7), and for the rest of the key enzymes, the presence/absence varies per species (Additional file 6). In total, of the 43 key enzymes present in $B$. cinerea, $B$. calthae and $B$. galanthina shared the highest number of orthologs (31 key enzymes), while $B$. paeoniae and $B$. porri shared the lowest number (24 key enzymes) with B. cinerea, respectively. In total, there are 17 SM key enzymes that are present in all Botrytis species, which is noticeably low, when considering that there are 16 SM key enzymes shared between the more distantly related S. sclerotiorum and B. cinerea (Fig. 6). The SM key enzymes that are shared between $S$. sclerotiorum and $B$. cinerea, and B. cinerea and the rest of the Botrytis spp. are not the same, and only 11 key enzymes overlap between all Botrytis species and S. sclerotiorum.

The result of this comparison differs from a previous study which reported that 19 SM key enzymes were shared between S. sclerotiorum and B. cinerea [24]. This difference results from improvements of the assemblies and annotations in each species [17, 36]. For instance, the gene sizes of $p k s 1$ and $p k s 18$, which were previously reported to be shared between $S$. sclerotiorum and $B$. cinerea, have changed considerably, around $10 \%$ in the case of Bcpks1, and 64 and $300 \%$, for Bcpks 18 and $S s p k s 18$, respectively. Also, Ssdmats1 which had homology to $B c d m a t s 1$, is now more closely related to Bcdmats2. Interestingly, the updated annotation of B. cinerea B05.10 no longer contains a gene that encodes the diterpene cyclase $B c d t c 2$, which highlights the importance of having a good genome assembly and annotation.

The distribution of SM key enzyme genes among the ten Botrytis species analysed here is very patchy. The chemical structure of metabolites produced by most Botrytis SM gene clusters remain to be characterized, and there is no information under which conditions these gene clusters are expressed. Altogether, it is difficult to pinpoint SM gene clusters that may be interesting candidates for functional studies that aim to identify host range determinants.

\section{Conclusions}

In this study we present the draft genome of nine species of Botrytis along with a comparative analysis with the previously sequenced $B$. cinerea. Our results show that overall Botrytis species share the main signatures and protein families in the secreted proteins. Furthermore, all effectors that were previously functionally characterized in $B$. cinerea are also present in other members of the genus. Analysis of the secondary metabolite biosynthetic gene clusters also gave insights in the complexity of presence/ absence of these clusters. These genome data will provide leads to design hypotheses about candidate host range determinants in the Botrytis genus, either in the effector repertoire or in genes that participate in the tolerance to antimicrobial compounds of the host plant species. In the present study we especially focused on Botrytis species infecting ornamental flower bulb crops that are dispersed through the phylogeny of the species. The analysis of genomes of additional Botrytis species that are phylogenetically more closely related to each other will enable to zoom in on a further characterization of secreted proteins with potential roles in the infection process. 


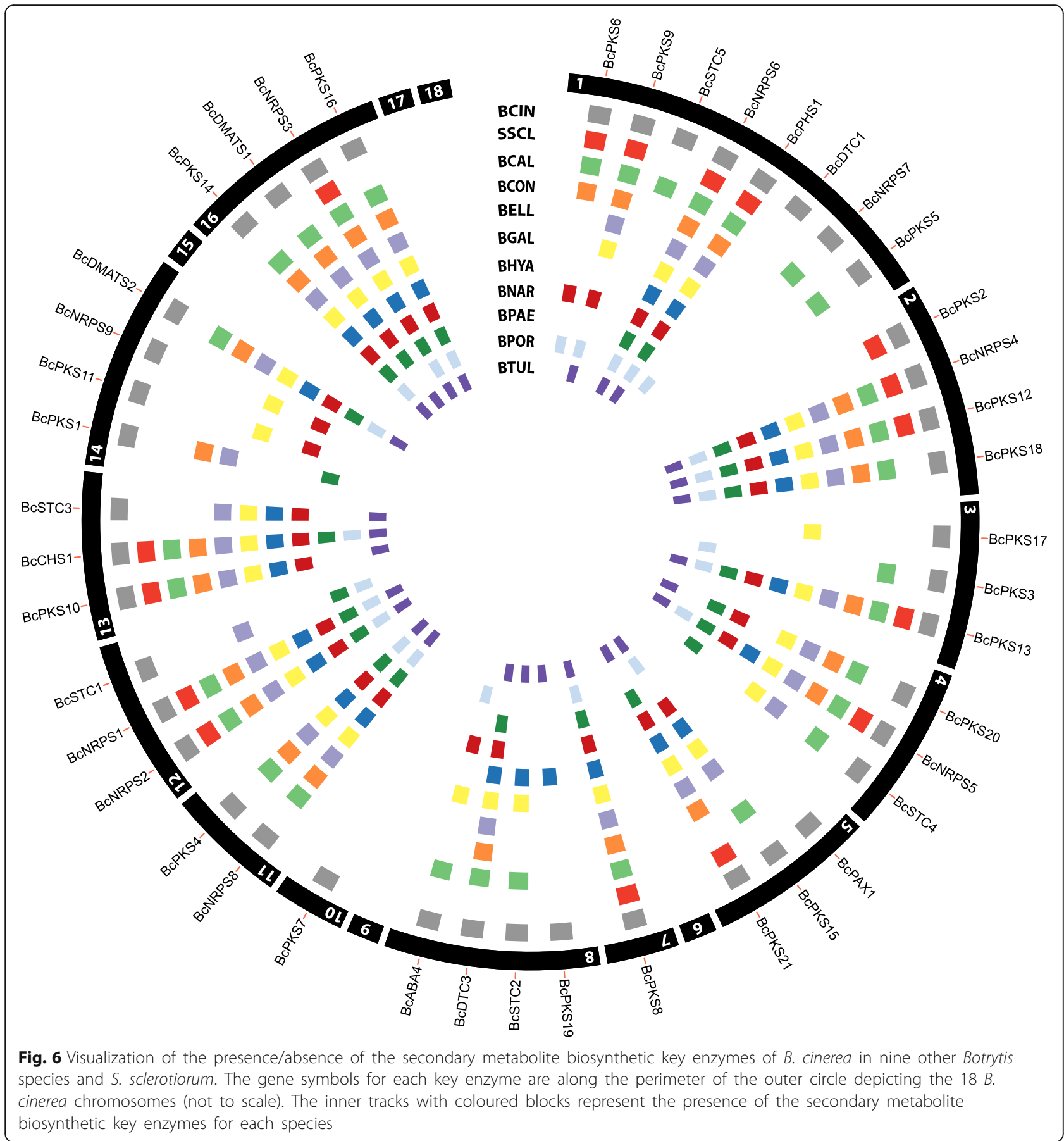

\section{Methods}

Strains and culture conditions

The fungal species used for this study are listed in Table 4. All fungal species were kept as conidial suspensions in $15 \%$ glycerol at $-80^{\circ} \mathrm{C}$ for long storage and grown on malt extract plates at $21^{\circ} \mathrm{C}$ before DNA extraction. DNA extraction was done as described by [46].

\section{Sequencing and assembly}

All sequences were sequenced with a paired-end library of $150 \mathrm{bp}$ insert size, with a read length of $2 \times 91 \mathrm{bp}$, except for B. tulipae, in which a paired-end library of $400 \mathrm{bp}$ insert size, and an additional mate pair library of $3.5 \mathrm{~kb}$ were constructed. DNA from B. tulipae was sequenced by Macrogen (Korea) while DNA from the other eight 
Table 4 Information about the strains used in this study

\begin{tabular}{llll}
\hline Botrytis sp. & Acronym & Isolate & Main host \\
\hline B. calthae & BCAL & MUCL2830 & Caltha pallustris \\
B. cinerea & BCIN & B05.10 & $>1000$ host species \\
B. convoluta & BCON & MUCL11595 & Iris \\
B. elliptica & BELL & Be9601 & Lilium \\
B. galanthina & BGAL & MUCL435 & Galanthus \\
B. hyacinthi & BHYA & Bh0001 & Hyacinthus \\
B. narcissicola & BNAR & M2120 & Narcissus \\
B. paeoniae & BPAE & Bp 0003 & Paeonia section paeoniae \\
B. porri & BPOR & MUCL3349 & Allium porrum \\
B. tulipae & BTUL & Bt9001 & Tulipa
\end{tabular}

species was sequenced by DNAVision (Liège, Belgium). De novo assembly was performed using the improved A5 pipeline [47], and further improved with Gapcloser from the Soapdenovo assembler [48]. For B. tulipae, assembly was performed using Spades assembler v.3.10.1 [49]. Completeness of the genome assemblies was assessed by the Benchmarking Universal Single-Copy Orthologs (BUSCO) v.2.0.1 software tool [50]. The GC content distribution of the sequenced genomes was examined using OcculterCut with default settings [19], and the analysis of repeat induced point mutations (RIP) was performed with RIPCAL [22].

\section{Genome annotation}

The assembled genomes were annotated using the MAKER (v.2.31.9) pipeline [51]. Before annotation, a species-specific repeat library was constructed using RepeatModeler (v.1.0.8) in order to mask repeats [52]. Gene models were predicted with AUGUSTUS [53], SNAP [54], and GeneMark-ES [55] ab initio gene predictors. The gene models of the manually curated genome of $B$. cinerea, and all the fungal proteins available in the Swissprot database were provided as evidence for gene prediction. The predicted proteins were functionally annotated using BLASTp [56] against the non-redundant database of the National Center of Biotechnology Information (NCBI) and classified by InterProScan and Pfam analysis [57].

\section{Phylogenetic and phylogenomic analysis}

The phylogenetic relationships were determined between all species sequenced, including the previously sequenced B. cinerea B05.10 [17], and using S. sclerotiorum [36] as the outgroup of the tree. The tree was constructed using 7668 single-copy orthologue genes, identified with Orthofinder [58]. The protein sequence for each gene was aligned and concatenated into a single matrix using MAFFT [59], and a maximum likehood phylogenetic tree was inferred with RAxML v.8.2.10 [60] using a generalized time reversible
(GTR) plus GAMMA amino acid substitution model with 100 rapid bootstraps. A pan-genome analysis was done to calculate the number of core genes and was estimated using OrthoMCL [61] implemented in GET_HOMOLOGUESEST [62] with e-value $1 \mathrm{e}^{-5}$ and $75 \%$ coverage. For the pangenome analysis, only the orthogroups present in at least two species were included.

\section{Secretome, GOterm and effector prediction}

Genes encoding putatively secreted proteins were identified for each Botrytis genome using several prediction tools. Signal-P v4.1 [63] was initially used to screen for a signal peptide, followed by TMHMM v.2.0 [64] to identify putative transmembrane domains. Proteins that did not have a signal peptide, or that had a transmembrane domain (a single transmembrane domain in the first 60 amino acids was allowed) were discarded. WoLF PSORT was used to predict protein localization [65]. Effectors were predicted using the EffectorP tool [66]. The GO enrichment in molecular functions was produced with the dcGO:database [67] and InterPro v66.0 [68]. To determine whether there was a relation between putative effector proteins and repeats, the distance to the nearest repeats was measured. Likewise, the distance between non-effector proteins and repeats was measured, using a random subset containing the same number of proteins as the set of effector proteins for that same species. The distances for the putative effector proteins were compared to the distances for the random non-effector proteins using Wilcoxon's test in $\mathrm{R}$ (version 3.5.2).

\section{Secondary metabolite analysis}

Identification of genes involved in the biosynthesis of secondary metabolites were based on homology to previously described genes in Botrytis cinerea and S. sclerotiorum [24, 44]. Sequences of the key enzymes were subjected to a BLASTn analysis to all Botrytis sp. genomes using an e-value of $1 \mathrm{e}^{-5}$ and a sequence identity of $70 \%$. Moreover, putative gene clusters that are predicted to be involved in biosynthesis of secondary metabolites were identified using antiSMASH (Antibiotics and Secondary Metabolite Analysis SHell) version 4.0.1 [69].

\section{Additional files}

Additional file 1: Percentage differences between dinucleotides frequencies of AT-rich regions and GC-equilibrated regions. A positive number indicate a higher value in AT-rich regions and a negative number indicate a higher value in GC-equilibrated regions. (XLSX $11 \mathrm{~kb}$ )

Additional file 2: Fold differences of the dinucleotide frequencies of Botrytis spp. repeat elements relative to the control, and estimation of RIP indices. (PDF $316 \mathrm{~kb}$ )

Additional file 3: Number of genes encoding secreted proteins in Botrytis species grouped by GO annotation for the Biological Process and Cellular Component domains. The average of all species is shown, error 
bars indicate the deviation in number of genes between the species. (PDF $20 \mathrm{~kb}$ )

Additional file 4: Distance to nearest repeat region of putative effectors and a random gene set of non-effectors. The scale in the $y$ axis is measured in $\mathrm{bp}$ to the nearest repeat. Asterisks represent different $P$ values $\left(^{*}=\right.$ $P<0.05 ;{ }^{* *}=P<0.001$; Wilcoxon's test). (PDF $169 \mathrm{~kb}$ )

Additional file 5: List of putative effector genes present in Botrytis spp. (XLSX $28 \mathrm{~kb})$

Additional file 6: Summary of the presence/absence of all described secondary metabolites key enzymes of Botrytis cinerea in Botrytis spp. (XLSX $13 \mathrm{~kb})$

\section{Abbreviations}

BUSCO: Benchmarking Universal Single-Copy Orthologs; G3PDH: Glyceraldehyde 3-phosphate dehydrogenase protein; GO: Gene Ontology; HSP60: Heat shock protein 60; PCWDE: Plant cell wall degrading enzyme; RIP: Repeat-Induced Point mutation; RPB2: RNA polymerase II protein; SM: Secondary Metabolites

\section{Acknowledgements}

The authors acknowledge the contribution of Alicia Abarca, Si Qin, Michele Malvestiti, Guido Puccetti, and Maikel Steentjes in the annotation of the nine genomes.

\section{Funding}

The research of Claudio A. Valero-Jiménez is supported by the Dutch Technology Foundation STW, which is part of the Netherlands Organisation for Scientific Research (NWO), and which is partly funded by the Ministry of Economic Affairs (project 15003). Javier Veloso was funded by the Postdoctoral program of the Xunta de Galicia (Spain). The funding organizations were neither involved in the design of the study and collection, analysis, and interpretation of data, nor in writing the manuscript.

\section{Availability of data and materials}

The Whole Genome Shotgun project has been deposited in GenBank under the Bioproject number PRJNA401386. The genomes are deposited under the genome accession numbers: PHWZ00000000 (B. calthae), PQXN00000000 (B. convoluta), PQXM00000000 (B. elliptica), PQXL00000000 (B. galanthina), PQXK00000000 (B.hyacinthi), PQXJ00000000 (B. narcissicola), PQXI00000000 (B. peaoniae), PQXO00000000 (B. porri), and PQXH00000000 (B. tulipae).

\section{Authors' contributions}

JVK and MS conceived the study. MS performed DNA extractions and performed preliminary assemblies. CAVJ performed the final assemblies and gene predictions. CAVJ, JV and JvK analyzed the data. CAVJ, JvK and JV prepared the figures and the manuscript. All authors read and approved the final manuscript.

\section{Ethics approval and consent to participate}

Not applicable

\section{Consent for publication}

Not applicable

\section{Competing interests}

The authors declare that they have no competing interests.

\section{Publisher's Note}

Springer Nature remains neutral with regard to jurisdictional claims in published maps and institutional affiliations.

\section{Author details}

'Laboratory of Phytopathology, Wageningen University, 6708PB Wageningen, the Netherlands. ${ }^{2}$ Department of Biology, Faculty of Sciences, University of A Coruña, A Coruña, Spain. ${ }^{3}$ Biosystematics Group, Wageningen University, 6708PB Wageningen, the Netherlands. ${ }^{4}$ Present address: RIKILT Wageningen University and Research, 6708WB Wageningen, the Netherlands.
Received: 31 August 2018 Accepted: 3 March 2019

Published online: 12 March 2019

\section{References}

1. Hyde KD, Nilsson RH, Alias SA, Ariyawansa HA, Blair JE, Cai L, et al. One stop shop: backbones trees for important phytopathogenic genera: I (2014). Fungal Divers. 2014;67:21-125.

2. Garfinkel AR, Lorenzini M, Zapparoli G, Chastagner GA. Botrytis euroamericana, a new species from peony and grape in North America and Europe. Mycologia. 2017;109:495-507. https://doi.org/10.1080/00275514.2017.1354169.

3. Grant-Downton RT, Terhem RB, Kapralov MV, Mehdi S, Rodriguez-Enriquez MJ, Gurr SJ, et al. A novel Botrytis species is associated with a newly emergent foliar disease in cultivated Hemerocallis. PLoS One. 2014;9:e0089272.

4. Elad Y, Pertot I, Cotes Prado AM, Stewart A. Plant hosts of Botrytis spp. In: Fillinger S, Elad Y, editors. Botrytis -- the fungus, the pathogen and its Management in Agricultural Systems. Cham: Springer International Publishing; 2016. p. 413-86. https://doi.org/10.1007/978-3-319-23371-0_20.

5. Staats M, van Baarlen P, van Kan JAL. Molecular phylogeny of the plant pathogenic genus Botrytis and the evolution of host specificity. Mol Biol Evol. 2005;22:333-46.

6. van Kan JAL. Licensed to kill: the lifestyle of a necrotrophic plant pathogen. Trends Plant Sci. 2006;11:247-53.

7. Vleeshouwers VGAA, Oliver RP. Effectors as tools in disease resistance breeding against biotrophic, hemibiotrophic, and necrotrophic plant pathogens. Mol Plant-Microbe Interact. 2014;27:196-206. https://doi.org/10. 1094/MPMI-10-13-0313-IA.

8. Breen S, Williams SJ, Winterberg B, Kobe B, Solomon PS. Wheat PR-1 proteins are targeted by necrotrophic pathogen effector proteins. Plant J. 2016;88:13-25.

9. Navarre D, Wolpert TJ. Inhibition of the glycine decarboxylase multienzyme complex by the host-selective toxin victorin. Plant Cell. 1995;7:463-71.

10. Condon BJ, Leng $Y$, Wu D, Bushley KE, Ohm R, Otillar R, et al. Comparative genome structure, secondary metabolite, and effector coding capacity across Cochliobolus pathogens. PLoS Genet. 2013;9: e1003233. https://doi.org/10.1371/journal.pgen.1003233.

11. Frías M, González C, Brito N. BcSpl1, a cerato-platanin family protein, contributes to Botrytis cinerea virulence and elicits the hypersensitive response in the host. New Phytol. 2011;192:483-95.

12. Schouten $A$, van Baarlen $P$, van Kan JAL. Phytotoxic Nep1-like proteins from the necrotrophic fungus Botrytis cinerea associate with membranes and the nucleus of plant cells. New Phytol. 2008;177:493-505.

13. Dalmais B, Schumacher J, Moraga J, Le Pêcheur P, Tudzynski B, Collado IG, et al. The Botrytis cinerea phytotoxin botcinic acid requires two polyketide synthases for production and has a redundant role in virulence with botrydial. Mol Plant Pathol. 2011;12:564-79.

14. Pinedo C, Wang C-M, Pradier J-M, Dalmais B, Choquer M, Le Pêcheur P, et al. Sesquiterpene synthase from the botrydial biosynthetic gene cluster of the phytopathogen Botrytis cinerea. ACS Chem Biol. 2008;3:791-801. https://doi.org/10.1021/cb800225v.

15. van Baarlen $P$, Staats M, van Kan JAL. Induction of programmed cell death in lily by the fungal pathogen Botrytis elliptica. Mol Plant Pathol. 2004;5:559-74.

16. Harrison JG. The production of toxins by Botrytis fabae in relation to growth of lesions on field bean leaves at different humidities. Ann Appl Biol. 1980; 95:63-71.

17. van Kan JAL, Stassen JHM, Mosbach A, van Der Lee TAJ, Faino L, Farmer AD, et al. A gapless genome sequence of the fungus Botrytis cinerea. Mol Plant Pathol. 2017;18:75-89. https://doi.org/10.1111/mpp.12384.

18. Dong S, Raffaele S, Kamoun S. The two-speed genomes of filamentous pathogens: waltz with plants. Curr Opin Genet Dev. 2015;35:57-65. https://doi.org/10.1016/j.gde.2015.09.001.

19. Testa AC, Oliver RP, Hane JK. OcculterCut: a comprehensive survey of ATrich regions in fungal genomes. Genome Biol Evol. 2016:8:2044-64.

20. Raffaele $S$, Kamoun S. Genome evolution in filamentous plant pathogens: why bigger can be better. Nat Rev Microbiol. 2012;10:417-30.

21. Chen YC, Liu T, Yu CH, Chiang TY, Hwang CC. Effects of GC bias in nextgeneration-sequencing data on de novo genome assembly. PLoS One. 2013;8:e62856.

22. Hane JK, Oliver RP. RIPCAL: a tool for alignment-based analysis of repeatinduced point mutations in fungal genomic sequences. BMC Bioinformatics. 2008;9(1):-12. 
23. Clutterbuck AJ. Genomic evidence of repeat-induced point mutation (RIP) in filamentous ascomycetes. Fungal Genet Biol. 2011;48:306-26.

24. Amselem J, Cuomo CA, van Kan JAL, Viaud M, Benito EP, Couloux A, et al. Genomic analysis of the necrotrophic fungal pathogens Sclerotinia sclerotiorum and Botrytis cinerea. PLoS Genet. 2011;7:e1002230.

25. Heard S, Brown NA, Hammond-Kosack K. An interspecies comparative analysis of the predicted secretomes of the necrotrophic plant pathogens Sclerotinia sclerotiorum and Botrytis cinerea. PLoS One. 2015;10:e0130534.

26. Brito N, Espino JJ, González C. The endo- $\beta-1,4-x y l a n a s e ~ X y n 11 A$ is required for virulence in Botrytis cinerea. Mol Plant-Microbe Interact. 2006;19:25-32.

27. Kars I, Krooshof GH, Wagemakers L, Joosten R, Benen JAE, van Kan JAL. Necrotizing activity of five Botrytis cinerea endopolygalacturonases produced in Pichia pastoris. Plant J. 2005;43:213-25.

28. ten Have A, Mulder W, Visser J, van Kan JAL. The endopolygalacturonase gene $B c p g 1$ is required for full virulence of Botrytis cinerea. Mol PlantMicrobe Interact. 1998;11:1009-16.

29. Gourgues $M$, Brunet-Simon A, Lebrun MH, Levis C. The tetraspanin BcPls1 is required for appressorium-mediated penetration of Botrytis cinerea into host plant leaves. Mol Microbiol. 2004:51:619-29.

30. Staats M, van Baarlen P, Schouten A, van Kan JAL, Bakker FT. Positive selection in phytotoxic protein-encoding genes of Botrytis species. Fungal Genet Biol. 2007:44:52-63.

31. Staats M, van Baarlen P, Schouten A, van Kan JAL. Functional analysis of NLP genes from Botrytis elliptica. Mol Plant Pathol. 2007;8:209-14.

32. Choi W, Dean RA. The adenylate cyclase gene MAC1 of Magnaporthe grisea controls appressorium formation and other aspects of growth and development. Plant Cell. 1997:9:1973-83.

33. DeZwaan TM, Carroll AM, Valent B, Sweigard JA. Magnaporthe grisea Pth11p is a novel plasma membrane protein that mediates appressorium differentiation in response to inductive substrate cues. Plant Cell. 1999;11:2013-30.

34. Kulkarni RD, Kelkar HS, Dean RA. An eight-cysteine-containing CFEM domain unique to a group of fungal membrane proteins. Trends Biochem Sci. 2003;28:118-21.

35. Zhu W, Wei W, Wu Y, Zhou Y, Peng F, Zhang S, et al. BcCFEM1, a CFEM domain-containing protein with putative GPI-anchored site, is involved in pathogenicity, conidial production, and stress tolerance in Botrytis cinerea. Front Microbiol. 2017;8:1807.

36. Derbyshire M, Denton-Giles M, Hegedus D, Seifbarghi S, Rollins J, van Kan J, et al. The complete genome sequence of the phytopathogenic fungus sclerotinia sclerotiorum reveals insights into the genome architecture of broad host range pathogens. Genome Biol Evol. 2017;9:593-618.

37. Sbaghi $M$, Jeandet $P$, Bessis $R$, Leroux P. Degradation of stilbene-type phytoalexins in relation to pathogenicity of Botrytis cinerea to grapevines. Plant Pathol. 1996:45:139-44.

38. Quidde T, Osbourn AE, Tudzynski P. Detoxification of a-tomatine by Botrytis cinerea. Physiol Mol Plant Pathol. 1998:52:151-65.

39. Schönbeck F, Schroeder C. Role of antimicrobial substances (tuliposides) in tulips attacked by Botrytis spp. Physiol Plant Pathol. 1972;2:91-9. https://doi.org/10.1016/0048-4059(72)90015-X.

40. Mansfield JW, Widdowson DA. The metabolism of wyerone acid (a phytoalexin from Vicia faba L.) by Botrytis fabae and B. cinerea. Physiol Plant Pathol. 1973;3:393-404.

41. Schouten A, Wagemakers CAM, Stefanato F, van der Kaaij RM, van Kan JAL. Resveratrol acts as natural profungicide: self-intoxication by a fungal laccase. Mol Microbiol. 2002:43:883-94.

42. Jarvis MC, Forsyth W, Duncan HJ. A survey of the pectic content of nonlignified monocot cell walls. Plant Physiol. 1988;88:309-14. https://doi.org/10.1104/pp.88.2.309.

43. Keller NP, Turner G, Bennett JW. Fungal secondary metabolism - from biochemistry to genomics. Nat Rev Microbiol. 2005;3:937-47. https://doi.org/ 10.1038/nrmicro1286.

44. Collado IG, Viaud M. Secondary metabolism in Botrytis cinerea: combining genomic and metabolomic approaches. In: Fillinger S, Elad $Y$, editors. Botrytis -- the fungus, the pathogen and its Management in Agricultural Systems. Cham: Springer International Publishing; 2016. p. 291-313. https://doi.org/10.1007/978-3-319-23371-0_15.

45. Renshaw JC, Robson GD, Trinci APJ, Wiebe MG, Livens FR, Collison D, et al. Fungal siderophores: structures, functions and applications. Mycol Res. 2002; 106:1123-42. https://doi.org/10.1017/S0953756202006548.

46. Staats M, van Kan JAL. Genome update of Botrytis cinerea strains B05.10 and T4. Eukaryot Cell. 2012;11:1413-4.
47. Coil D, Jospin G, Darling AE. A5-miseq: an updated pipeline to assemble microbial genomes from Illumina MiSeq data. Bioinformatics. 2015;31:587-9.

48. Luo R, Liu B, Xie Y, Li Z, Huang W, Yuan J, et al. SOAPdenovo2: an empirically improved memory-efficient short-read de novo assembler. Gigascience. 2012;1:18. https://doi.org/10.1186/2047-217X-1-18.

49. Bankevich A, Nurk S, Antipov D, Gurevich AA, Dvorkin M, Kulikov AS, et al. SPAdes: a new genome assembly algorithm and its applications to singlecell sequencing. J Comput Biol. 2012;19:455-77.

50. Simão FA, Waterhouse RM, loannidis P, Kriventseva EV, Zdobnov EM. BUSCO: assessing genome assembly and annotation completeness with single-copy orthologs. Bioinformatics. 2015;31:3210-2.

51. Campbell MS, Holt C, Moore B, Yandell M. Genome annotation and curation using MAKER and MAKER-P. In: Current Protocols in Bioinformatics. Hoboken, NJ: John Wiley \& Sons, Inc.; 2014. p. 4.11.1-4.11.39. https://doi.org/ 10.1002/0471250953.bi0411s48.

52. Tempel S. Using and understanding RepeatMasker. In: Bigot Y, editor. Mobile genetic elements: protocols and genomic applications. Totowa, NJ: Humana Press; 2012. p. 29-51. https://doi.org/10.1007/978-1-61779-603-6_2.

53. Stanke M, Schöffmann O, Morgenstern B, Waack S. Gene prediction in eukaryotes with a generalized hidden Markov model that uses hints from external sources. BMC Bioinformatics. 2006;7:62. https://doi.org/10.1186/ 1471-2105-7-62.

54. Korf I. Gene finding in novel genomes. BMC Bioinformatics. 2004;5:59.

55. Ter-Hovhannisyan V, Lomsadze A, Chernoff YO, Borodovsky M. Gene prediction in novel fungal genomes using an ab initio algorithm with unsupervised training. Genome Res. 2008;18:1979-90.

56. Altschul SF, Gish W, Miller W, Myers EW, Lipman DJ. Basic local alignment search tool. J Mol Biol. 1990;215:403-10. https://doi.org/10.1016/S00222836(05)80360-2.

57. Jones P, Binns D, Chang HY, Fraser M, Li W, McAnulla C, et al. InterProScan 5: genome-scale protein function classification. Bioinformatics. 2014;30: $1236-40$.

58. Emms DM, Kelly S. OrthoFinder: solving fundamental biases in whole genome comparisons dramatically improves orthogroup inference accuracy. Genome Biol. 2015;16:157. https://doi.org/10.1186/s13059-015-0721-2.

59. Katoh K, Misawa K, Kuma K, Miyata T. MAFFT: a novel method for rapid multiple sequence alignment based on fast Fourier transform. Nucleic Acids Res. 2002;30:3059-66.

60. Stamatakis A. RAxML version 8: a tool for phylogenetic analysis and postanalysis of large phylogenies. Bioinformatics. 2014;30:1312-3.

61. Li L. OrthoMCL: identification of ortholog groups for eukaryotic genomes. Genome Res. 2003;13:2178-89. https://doi.org/10.1101/gr.1224503.

62. Contreras-Moreira B, Vinuesa P. GET_HOMOLOGUES, a versatile software package for scalable and robust microbial pangenome analysis. Appl Environ Microbiol. 2013;79:7696-701.

63. Petersen TN, Brunak S, von Heijne G, Nielsen H. SignalP 4.0: discriminating signal peptides from transmembrane regions. Nat Methods. 2011:8:785-6. https://doi.org/10.1038/nmeth.1701.

64. Krogh A, Larsson B, von Heijne G, Sonnhammer EL. Predicting transmembrane protein topology with a hidden markov model: application to complete genomes. J Mol Biol. 2001;305:567-80. https://doi.org/10.1006/ jmbi.2000.4315.

65. Horton P, Park KJ, Obayashi T, Fujita N, Harada H, Adams-Collier CJ, et al. WoLF PSORT: protein localization predictor. Nucleic Acids Res 2007;35 SUPPL.2:585-587.

66. Sperschneider J, Gardiner DM, Dodds PN, Tini F, Covarelli L, Singh KB, et al. EffectorP: predicting fungal effector proteins from secretomes using machine learning. New Phytol. 2016;210:743-61.

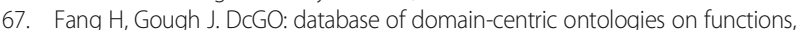
phenotypes, diseases and more. Nucleic Acids Res. 2013;41:D536-44.

68. Finn RD, Attwood TK, Babbitt PC, Bateman A, Bork P, Bridge AJ, et al. InterPro in 2017-beyond protein family and domain annotations. Nucleic Acids Res. 2017;45:D190-9.

69. Weber T, Blin K, Duddela S, Krug D, Kim HU, Bruccoleri R, et al. antiSMASH 3.0-a comprehensive resource for the genome mining of biosynthetic gene clusters. Nucleic Acids Res. 2015;43:W237-43. https://doi.org/10.1093/nar/gkv437. 